
allemande

50-2 | 2018

Humanités environnementales - Quoi de neuf du côté des méthodes?

\title{
Bâtir les humanités environnementales des matériaux géo-sourcés : construction et déconstruction des passerelles franco-germaniques
}

\section{Sophie Némoz}

\author{
(2) OpenEdition \\ Journals \\ Édition électronique \\ URL : https://journals.openedition.org/allemagne/965 \\ DOI : 10.4000/allemagne.965 \\ ISSN : 2605-7913 \\ Éditeur \\ Société d'études allemandes \\ Édition imprimée \\ Date de publication : 30 décembre 2018 \\ Pagination : 377-392 \\ ISSN : 0035-0974 \\ Référence électronique \\ Sophie Némoz, «Bâtir les humanités environnementales des matériaux géo-sourcés : construction et \\ déconstruction des passerelles franco-germaniques ", Revue d'Allemagne et des pays de langue \\ allemande [En ligne], 50-2 | 2018, mis en ligne le 30 décembre 2019, consulté le 03 juin 2021. URL \\ http://journals.openedition.org/allemagne/965; DOI : https://doi.org/10.4000/allemagne.965
}




\section{Bâtir les humanités environnementales des matériaux géo-sourcés: construction et déconstruction des passerelles franco-germaniques}

\section{- Sophie Némoz*}

«Un mur de pisé s'effondre sur un homme». Dans le flux quasi-continu des multiples sites d'informations de nos sociétés contemporaines, ce titre publié il y a quatre ans sur Internet, par la presse locale, via ledauphine.com, a été repris par le journal télévisé France 3 Auvergne-Rhône-Alpes. Ce média régional avait pu mettre en avant une première explication relevant des sciences des matériaux:

«Un phénomène qui n’est pas si rare que ça dans le Dauphiné. Le pisé est un matériau utilisé en construction depuis des siècles et fait partie du patrimoine local. Mais l'humidité révèle parfois les faiblesses de ces murs en terre crue.»

De fait, avant cet incident survenu au mois de juin 2014, de tels événements ont été signalés comme des accidents domestiques sur les territoires ruraux du nord-Isère. Ainsi, à Flachères, une commune de 500 habitants, un père de famille, quinquagénaire, originaire du nord de la France, est enterré vivant sous l'effondrement d'un mur de pisé à l'intérieur d'une grange et ce, alors qu'il entreprenait « un vaste chantier de réhabilitation de la bâtisse en deux habitations sur $180 \mathrm{~m}^{2}{ }^{(1)}$. Ce qui se lit dans la rubrique «des faits divers» peut être révélateur de logiques plus profondes, d'un «fait social total». Marcel Mauss $^{(2)}$ en a forgé le concept et l'outil, le principe est de mettre au jour des systèmes sociaux entiers, en examinant les multiples dimensions (sociologique, historique, économique, géographique, philosophique, politique...) dans une perspective relationnelle.

Notre article propose de poursuivre cette approche qui privilégie la pluridisciplinarité plusieurs années après la soutenance d'une thèse en sociologie sur les politiques,

* Maître de conférences en sociologie-anthropologie, Université de Bourgogne/Franche-Comté, UFR Sciences du langage de l'Homme et de la société.

1 Source: ledauphine.com, consulté le 25 juillet 2014.

2 Marcel Mauss, Essai sur le don. Forme et raison de l'échange dans les sociétés archaïques (1950), Paris, Presses universitaires de France (coll. Quadrige), 2007. 
ainsi que sur les pratiques professionnelles et habitantes d'un logement dit «durable ${ }^{(3)}$. À partir d'un échantillon qualitatif de près d'une centaine d'individus qui donnent eux-mêmes sens à leurs pratiques, la recherche les restitue à travers des descriptions fines des expériences, rendant compte à la fois du contexte, du cadre contraignant, normatif, pesant sur la situation, et des ressources que les acteurs mobilisent comme des significations qu'ils expriment dans des mots qui accompagnent leur pratique, dans des attitudes d'engagement et des signes des sentiments éprouvés. Lorsqu'un de nos enquêtés nous a annoncé le décès d'un auto-constructeur dans des circonstances similaires sur un des terrains français de notre ethnographie multi-sites, les caractères particuliers, exceptionnels et contingents de ce cas pour le moins localisé ont pu être interprétés comme une épreuve microsociale, à hauteur d'hommes et à l'échelle de leurs vies, un indice dramatique de l'ambivalence des processus d'innovation à l'œuvre. Une tension entre l'auto-destruction et la réalisation de soi nous a été livrée par les habitants rencontrés et ce, en nous exprimant immanquablement un système de représentations à travers lesquelles les individus se construisent, se réhabilitent d'un point de vue physique comme symbolique et prennent des risques jusqu'au péril de leur vie. Prenant acte des singularités, divers travaux sociologiques ${ }^{(4)}$ ont œuvré à une récente actualisation au sein de la discipline et des sciences humaines.

Que dit la répétition de ces éboulements de murs en terre? Ne faut-il pas les envisager comme la séquence d'une totalité dynamique plus vaste? Dans quelle mesure l'attention portée à leur matérialité permet-elle d'aiguiser un regard réflexif et historique sur l'imbrication des domaines généralement scindés par nos catégorisations? Qualifiée de "géo-sourcée», la figure de la terre comme matériau de construction peutelle contribuer à repenser le dualisme entre nature et société? Avec quelles fondations et vers quelles passerelles ce changement de perspective est-il susceptible d'enrichir l'ensemble des humanités environnementales?

Afin d'apporter des réponses à ce questionnement, nous invitons les lecteurs à faire connaissance avec les chantiers de réhabilitation de l'habitat en terre où nous avons initié, il y a plus de dix ans, une enquête ethnographique. Entendu comme un temps prolongé au cœur du terrain, ce parti pris méthodologique est ici ancré dans une perspective de long terme au cours de laquelle la technique de la revisite a été mobilisée en vue de comprendre et d'expliquer les variations ayant lieu au fur et à mesure du temps ${ }^{(5)}$. Partant de ces rencontres renouvelées à une décennie d'intervalle, une large part du matériel d'analyse a été recueillie à l'occasion des entretiens biographiques, des relevés photographiques et des observations retranscrites dans nos carnets d'enquête

3 Sophie Némoz, L' «éco-logis»: une innovation durable... Analyse sociologique de l'écologie résidentielle en France et au détour de la Finlande et l'Espagne, thèse de doctorat, Université Paris Descartes Sorbonne, 2009.

4 Danilo Martuccelli, La société singulariste, Paris, Armand Colin, 2010; Bruno Latour, Changer de société, refaire de la sociologie (2005), Paris, La Découverte, 2007.

5 La revisite des terrains d'enquêtes a bénéficié du soutien du Service de recherche de l'Université de Franche-Comté dans le cadre de deux projets lauréats des appels à recherches émergentes CRYSALIDE: Sophie Némoz (dir.), «L'habitat durable à l'épreuve des espaces et des temps sociaux», CRYSALIDE 2017-UFC, Maison des sciences de l'Homme et de l'environnement (MSHE Ledoux, USR 3124) et Sophie Némoz (dir.), «Revisiter les écologies de l’habiter comme une mosaïque sociale», CRYSALIDE 2018-UFC, MSHE Ledoux, USR 3124. 
de 2006 à 2018. Ce corpus vise à étudier dans un premier temps la manière dont le gros œuvre de la terre se pratique en France pour l'habitation contemporaine en milieu rural. En questionnant tout autant les qualifications que les disqualifications, les revers, à commencer par des effondrements répétitifs parfois mortels, les dispositifs de gouvernementalité pourront ensuite être replacés dans une temporalité relativement longue et une localité étendue par les relations transfrontalières avec les pays de langue allemande. Finalement, il y a là un espace de discussion des circuits de connaissances que ce texte non seulement introduit mais également investit par l'articulation des savoirs de pedigrees très divers que suscite l'environnement au sein, entre et par-delà les disciplines scientifiques.

\section{Découvrir le gros œuvre de la terre par la recherche ethnographique sur l'habitat dit durable}

À plusieurs dizaines de kilomètres des agglomérations de Lyon, de Grenoble ou de Chambéry, des espaces de rénovation de l'habitat vernaculaire en milieu rural peuvent paraître situés aux antipodes de l'impératif de densification lisible dans les textes institutionnels du développement urbain durable. Les travaux réalisés manuellement avec des matériaux ancestraux, des outils traditionnels, et peu de moyens mécaniques, vont aussi à l'encontre d'une «technicisation de l'écologie» telle que les comparaisons européennes ont pu l'observer dans le secteur du bâtiment ${ }^{(6)}$. Si la prédominance des enjeux de performance énergétique peut expliquer la généralisation de ce phénomène, les directives successives de l'Union européenne contribuant à définir un «format » à bâtir pour lutter contre les émissions de gaz à effet de serre et les dépenses d'énergie ${ }^{(7)}$, l'étude d'opérations "sur mesure», "au cas par cas», apporte un éclairage nouveau sur l'évolution et l'uniformisation des modes d'aménagement. L'effet de cette standardisation n'est effectivement pas sans réplique. Il suscite la reproduction d'un modèle d'habitat ancien que l'on peut désigner comme «alternatif» car aux fondements d'une critique et d'une action sociale collective:

«Pour tout te dire, je ne suis pas mécontent que tu cherches à savoir ce qui nous gêne dans l'idée de ville durable et sa politique... En ce moment, c'est tellement présenté comme la solution miracle qu'on n'a pas si souvent l'occasion d'en parler! D'abord, pour éviter tout malentendu, je préfère te dire que nous aussi, on se préoccupe de l'énergie, mais aussi de l'eau, de la santé, du positionnement du bâtiment par rapport à l'environnement [...] Mais ce qui nous paraît le plus grave, c'est la réglementation de la certification environnementale des matériaux qui va avec, et qui coûte aussi les yeux de la tête! Elle te demande de faire une batterie de tests de chaque composant. D'ailleurs, j'ai encore les papiers, je vais te montrer... [Cinq minutes plus tard] Donc, comme tu peux le voir, la certification exige un listing de tous les composants du matériau. Alors ça marche sûrement très bien pour les produits industriels mais, pour ce qui est des matières naturelles, tu ne trouves jamais deux fois la même composition et il te faut varier tes façons de faire! C'est complètement inadapté et

6 Simon Guy, Steven A. Moore (éd.), Sustainable Architecture. Cultures and Natures in Europe and North America, New York, Taylor \& Francis, 2005; S. Némoz, L'«éco-logis»: une innovation durable (note 3).

7 Sophie Némoz, «L'“éco-logis” politique: un dépaysement critique de l'habitat durable en Europe», Sciences de la société, « Habitat durable: approches critiques», nº 98 (2016), p. 31-43. 
disproportionné par rapport aux murs en adobe qu'on est quelques-uns à mettre en œuvre chez des particuliers. Surtout que les dépenses d'énergie, les émissions de $\mathrm{CO}_{2}$ depuis l'extraction de la terre jusqu'à la destruction, sont quasi nulles et on n'a pas besoin de faire Polytechnique pour s'en rendre compte! Une matière locale accessible, économique, peu énergivore, sans déchet ultime et appropriable par les usagers, c'est du bon sens mais pas dans la logique des Documents Techniques Unifiés! ${ }^{\left({ }^{(8)}\right.}$.

Figure 1. Matériel d'un atelier de construction en terre sur un chantier de réhabilitation d'une ancienne ferme
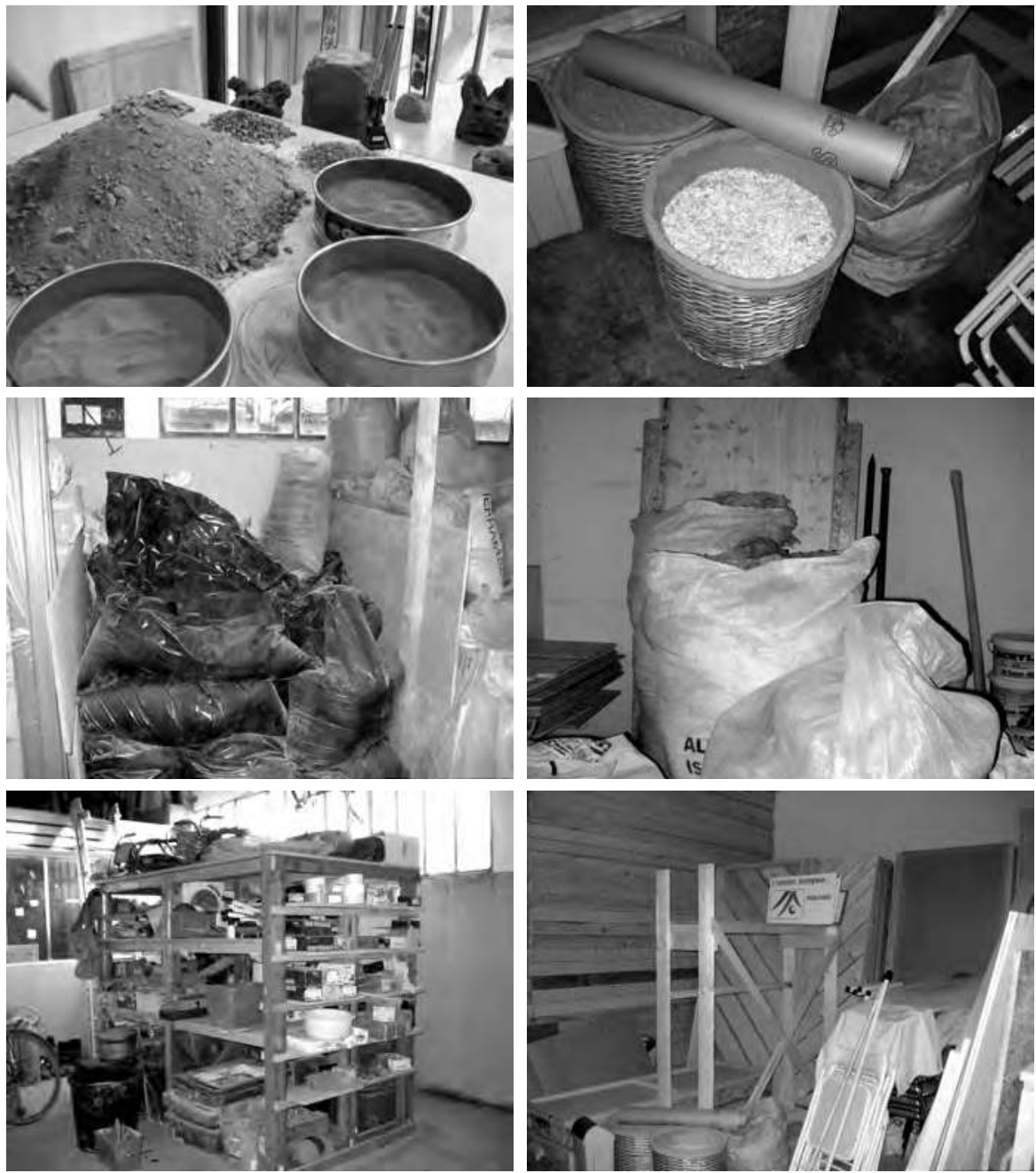

Sophie Némoz, 2009

8 Extrait d'un entretien réalisé en décembre 2006 par Sophie Némoz auprès d'un architecte, âgé de 34 ans, maître d'œuvre et constructeur de gros œuvre en terre crue dans le Dauphiné. 
Accompagnés d'une prise de vue systématique, les propos recueillis auprès d'un membre de ce petit nombre de professionnels de l'architecture et de la construction, engagés dans la réhabilitation écologique de l'habitat en terre des campagnes de l'Isère, pointent les formes de rationalisation perçues et l'imposition de normes associées au développement urbain durable. Les caractéristiques décrites renvoient à une forme de standardisation techniciste qui ne domine pas seulement l'urbanisme mais aussi la matérialité des bâtiments. Une tension ressort entre la normativité de ces standards internationaux qui sont à documenter selon des procédures nationales et la biodiversité contingente des matières premières locales avec lesquelles maîtres d'œuvre et mains-d'œuvre doivent moduler (figure 2).

Figure 2. Préparation de la mise en œuvre de la terre sur un chantier de réhabilitation d'une ancienne ferme
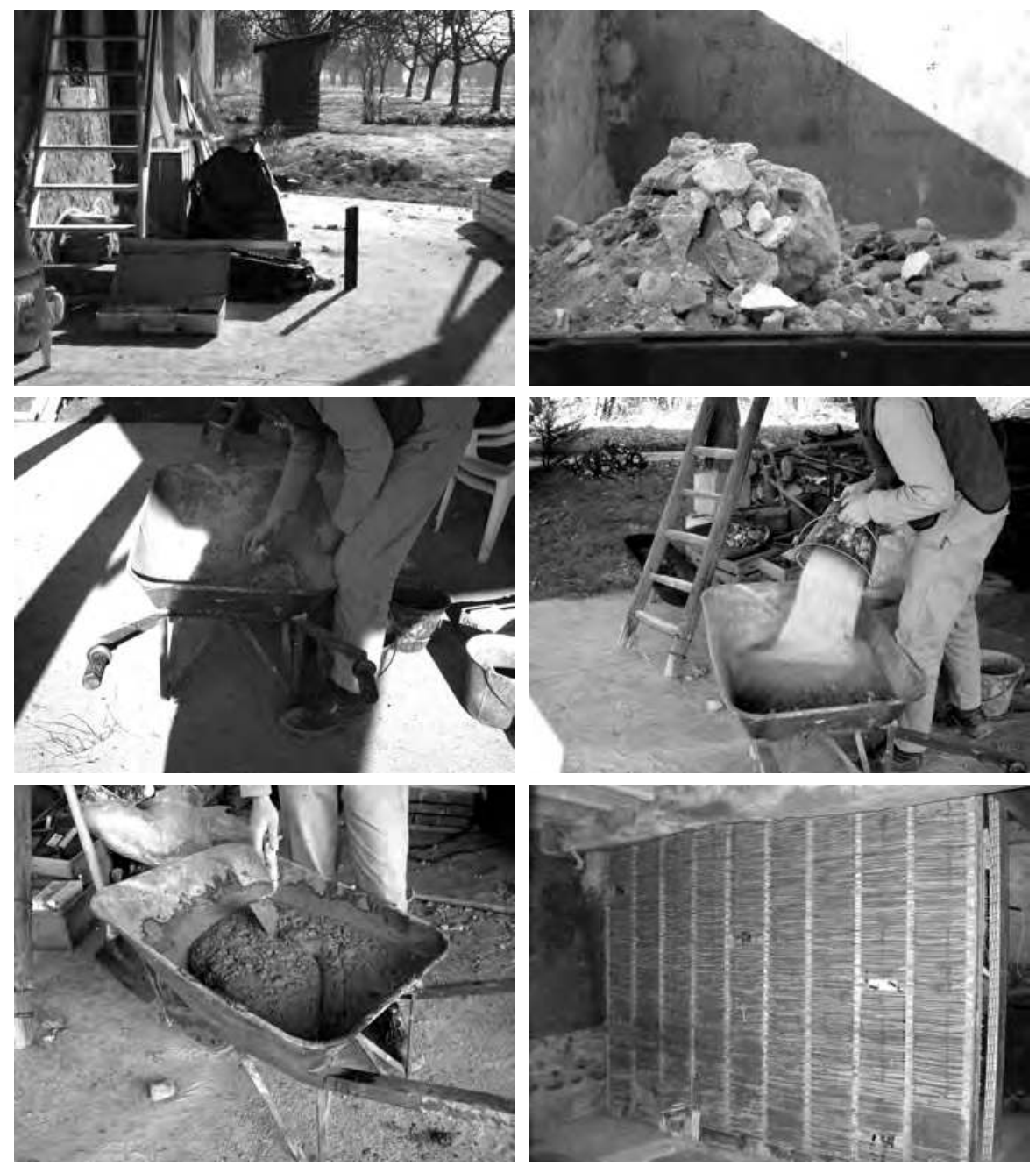

Sophie Némoz, 2009 
Par ailleurs, nos observations des chantiers nous ont appris que les maîtres d'œuvre ont à négocier étroitement avec les ménages propriétaires et maîtres d'ouvrage de la réhabilitation d'anciennes fermes en maisons individuelles. Si cette approche du projet se démarque du métier d'architecte professionnel et technique, comme de l'ouvrier exécutant dans le secteur du bâtiment, elle prend sens au regard du «bien singulier» ${ }^{(9)}$ que nous ont présenté les "particuliers» comme ces professionnels appellent leurs clients, dont les goûts et les exigences ne se limitent pas à des propriétés techniques:

"Vous savez quand on a hérité de cette vieille ferme, on ne connaissait rien de la construction en terre. Tout ce dont on était sûr, c'est qu'on ne voulait pas lui enlever son originalité, ses couleurs, sa forme, son charme, ça me ramène à tellement de choses, ce sont des images d'enfance pour moi, des souvenirs de famille, et en même temps, des aménagements devaient être faits pour pouvoir l'habiter et ne plus passer les week-ends dans la tente du jardin [...] À mes yeux, cette maison de campagne correspond tout à fait à l'idée d'habitat durable, de la préservation de la nature en vivant à son contact, en profitant de ses fruits tout en plantant de nouveaux arbres pour les récoltes à venir... Les anciens possédaient toutes ces connaissances dont on a complètement perdu la notion en ville. À Grenoble, on vivait en immeuble, on n’a jamais pu espérer avoir un tel logement! Avec cette maison, il a fallu s'éloigner de notre travail, de nos amis, des copains d'école des enfants... Et je ne vous dis pas le nombre d'insomnies que j'ai pu faire au moment où on recherchait des entreprises pour faire les travaux... Mon mari avait fait les plans, imaginé les réagencements à faire pour y vivre. Je pensais trouver des professionnels au village ou dans les environs car vu le nombre de maisons en pisé, les maçons d'ici devaient avoir l'habitude de travailler avec la terre et les matériaux rustiques. Je suis tombée de haut! Ils m’ont presque ri au nez quand je leur ai demandé s'il était possible d'utiliser ces mêmes techniques écologiques. Quand j'ai fini par trouver des spécialistes après avoir élargi mes recherches dans la région, il a fallu qu'on mette en sourdine nos grandes idées de participation au développement local! Vu le montant des devis et notre petit budget, les décisions n'ont pas été faciles à prendre. Pour la qualité du gros œuvre, le respect de l'environnement, on leur a fait entièrement confiance, mais pour reprendre le dessin de notre projet dans son ensemble, on a préféré leur dire à quoi s'attendre. Les finitions qu'ils avaient envisagées seront probablement confiées à nos ados pendant les vacances d'été! » ${ }^{(10)}$.

Entendue comme individuelle et comme originale, l'emprise du singulier se déploie dans ce long extrait d'entretien et à travers bien d'autres paroles recueillies auprès des ménages habitant des réhabilitations dites écologiques dans les campagnes du Dauphiné. Un maximum de valeurs symboliques et d'incertitudes sur la qualité ont été rattachées à ce type d'habitat faiblement concurrencé par les prix, donnant à voir les différentes caractéristiques d'une «économie des singularités » telle que le sociologue Lucien Karpik ${ }^{(11)}$ l'a conceptualisée à l'égard d'autres biens. Cela étant, les récits des propriétaires de ce type de biens résidentiels décrivent moins un artisanat local qu'un «fait social total».

9 Lucien KaRPiK, L'économie des singularités, Paris, Gallimard, 2007.

10 Extrait d'un entretien réalisé en mai 2007 par Sophie Némoz auprès d'une mère de famille, âgée de 50 ans, animatrice auprès de personnes handicapées et maître d'ouvrage de la rénovation d'une ancienne ferme en résidence principale dans un hameau isérois. 


\section{Revisiter les circuits de matériaux géo-sourcés comme une totalité transfrontalière}

"Dans ces phénomènes sociaux "totaux", comme nous proposons de les appeler, s'expriment à la fois et d'un coup toutes sortes d'institutions [...]. Au fond, corps, âme, société, tout ici se mêle. Ce ne sont plus des faits spéciaux de telle ou telle partie de la mentalité, ce sont des faits d'un ordre très complexe, le plus complexe imaginable, qui nous intéressent. C'est ce que je suppose d'appeler des phénomènes de totalité où prend part non seulement le groupe, mais encore, par lui, toutes les personnalités, tous les individus dans leur intégrité morale, sociale, mentale, et surtout, corporelle ou matérielle» ${ }^{(12)}$.

Ces différentes dimensions nous ont été signifiées par les habitants rencontrés, en nous faisant part d'un idéal de réalisation de soi à travers la rénovation écologique d'une ancienne bâtisse au milieu des champs. Dès nos premières visites il y a dix ans, nous avons découvert "des espaces pleins de temps", au sens où "ils permettent à certains caractères narratifs d'opérer dans la vie quotidienne » ${ }^{(13)}$. Plus qu'un retour au passé, les discours recueillis auprès des habitants et des professionnels des réhabilitations de maisons en pisé se sont positionnés de manière réactive et hors norme face aux conventions de la "ville durable». Au cours des entretiens, leur singularité s'est affirmée de façon explicite vis-à-vis des standards environnementaux de technisation contemporaine et de densification urbaine. Ils y répliquent par une écologie des pratiques anticonformistes, pour ne pas dire diamétralement opposées, en s'engageant dans la réhabilitation de l'habitat vernaculaire en milieu rural à l'aide des techniques ancestrales et peu industrialisées. Les spatialités, les temporalités, le rapport à la technique, à l'environnement, ou encore l'organisation sociale des aménagements bâtis et des processus de production comme de transmission marchande ou non marchande, se distinguent tant dans la géographie, la morphologie, l'esthétique que du point de vue de l'économie, de la sociologie ou des cadres juridiques. En urbanisme, ils ne sont pas sans faire penser à deux polarités extrêmes des théories et des doctrines, l'une dite "progressiste» en référence à une modernité fonctionnaliste et l'autre «culturaliste » ${ }^{(14)}$, faisant écho aux figures de la nostalgie. De là à conclure à l'existence de deux modèles urbanistiques, cela semble excessif. Si la question se pose au sujet de la "ville durable» ${ }^{(15)}$, ce ne sont pas tellement les «images de la ville future» ${ }^{(16)}$ qui préoccupent les professionnels et les habitants rencontrés. Ils se sont révélés plus soucieux de leurs capacités d'ajustement à la marge, des tactiques pour reprendre les mots de Michel de Certeau:

«[...] j'appelle tactique l'action calculée que détermine l'absence d'un propre. [...] La tactique n'a pour lieu que celui de l'autre. Aussi doit-elle jouer avec le terrain qui lui est imposé tel que l'organise la loi d'une force étrangère. Elle n'a pas le moyen de se tenir en elle-même, à distance, dans une position de retrait, de prévision et de rassemblement de soi: elle est mouvement “à l'intérieur du champ de vision de l'ennemi” [...] et de l'espace contrôlé par lui. Elle n'a donc pas le moyen de se donner un projet global ni de totaliser l'adversaire dans

12 Marcel Mauss, Sociologie et anthropologie, Paris, Presses universitaires de France (coll. Quadrige), 2003, p. 147 et 303.

13 Richard Sennet, La conscience de l'oil. Urbanisme et société (1992), Paris, Éditions de la Passion, 2000.

14 Françoise Choay, L'Urbanisme: utopies et réalités, Paris, Seuil, 1965.

15 Claire Carriou, Olivier Ratouis, «Quels modèles pour l'urbanisme durable?», Métropolitiques, 2014, https://www.metropolitiques.eu/Quelsmodeles-pour-l-urbanisme.html.

16 F. CHоAу, L'Urbanisme: utopies et réalités (note 14). 
un espace distinct, visible et objectivable. Elle fait du coup par coup. Elle profite des "occasions" et elle en dépend, sans base où stocker les bénéfices, augmenter un propre ou prévoir des sorties. Ce qu'elle gagne ne se garde pas. Ce non-lieu lui permet sans doute la mobilité, mais dans une docilité aux aléas du temps, pour saisir au vol les possibilités qu'offre un instant. Il lui faut utiliser, vigilante, les failles que les conjonctures particulières ouvrent dans la surveillance du pouvoir propriétaire. Elle y braconne. Elle y crée des surprises. Il lui est possible d'être là où on ne l'attend pas. Elle est ruse» ${ }^{(17)}$.

La réhabilitation écologique d’anciennes fermes en pisé résulte de trajectoires variées et peu anticipées de la part des professionnels et des habitants. Celles-ci sont indexées sur les situations, que ce soit la hausse des prix du foncier dans les agglomérations urbaines qui limite le choix des emplacements résidentiels vers des secteurs éloignés et mal desservis par les services, ou l'ouvrage privé de maisons individuelles. C'est une architecture dite mineure, que de jeunes diplômés de la profession, comme des cadres et des ouvriers en reconversion, ont investi en tant que maîtres d'œuvre et mains-d'œuvre, avec des matériaux et des techniques sans assurance professionnelle à défaut de certification et, en zone rurale, à la périphérie des plans d'urbanisme. L'effectivité sociale des singularités de la rénovation écologique de l'habitat rural est mise en exergue par les acteurs au regard des épreuves de relégations professionnelles et résidentielles qu'ils ont traversées et auxquelles ils se sont systématiquement reportés dans leurs récits.

L'enquête ethnographique permet de comprendre comment leurs pratiques singulières procèdent de tactiques, d'ajustements en situations de disqualifications face au renforcement des dispositifs de contrôle et de standardisation de la ville durable et des processus de production. Il y a là des phénomènes sociaux totaux jusqu'à en être mortels. Les tactiques n'anticipent pas, elles se définissent dans l'action, au coup par coup, par répliques, à travers la reproduction dite écologique d'un modèle ancien d'habitation rurale et face aux risques d'effondrements des parois en terre. Si le mouvement se concrétise par des actes créateurs et destructeurs, il n'en est pas moins porteur de sens lorsqu'on analyse chaque réplique comme une réponse, une expérience à la fois concrète et complexe, indissociable d'une totalité historique et territoriale plus vaste. En déployant la technique socio-anthropologique de la revisite, l'exercice de l'enquête réitérée sur la durée montre comment les coopérations de proximité se font et se défont au fur et à mesure du temps et des interactions avec des organismes, des organisations et des environnements non uniquement naturels mais aussi matériels, culturels, politiques et sociaux.

Dix ans après avoir observé le travail des acteurs qui étaient aux avant-postes du gros œuvre de la terre en France, la composition des liens et des lieux de contacts germaniques s'avère mouvante. D’une décennie à l'autre, la géographie des déplacements des personnes, des idées et des matériaux enquêtés trace des circuits de plus grande distance et fréquence entre les terrains français et les pays de langue allemande. Tout se passe comme si les importations des matériaux germaniques par camions remplis de sacs de terre avaient succédé aux séjours de formation auprès de leurs homologues des «autres pays», parmi lesquels l'Allemagne était systématiquement (et souvent uniquement) mentionnée ${ }^{(18)}$. Plusieurs millions de bâtiments en terre ont été construits en

17 Michel de Certeau, L'invention du quotidien, 1. Arts de faire, Paris, Gallimard, 1990, p. 60-61.

18 Anahita Grisoni, Sophie Némoz, «Les mouvements sociaux écologistes: entre réforme de soi et rapports de classe, entre histoires nationales et circulations européennes ", Socio-logos (en ligne), $\mathrm{n}^{\circ} 12$ (2017), http://socio-logos.revues.org/3145. 
Europe, non uniquement dans les territoires alémaniques, que ce soit au Danemark, en Suède, dans les pays de l'Est, ou encore en Espagne, en Grande-Bretagne, en Belgique, ou en France ${ }^{(19)}$. Si l'architecte François Cointeraux est «une grande figure du patrimoine régional Rhône-Alpes», "pionnier de la construction moderne en pisé» (20) aux XVIII ${ }^{\mathrm{e}}$ et XIX ${ }^{\mathrm{e}}$ siècles, aucun des praticiens rencontrés localement n'ont cité ce professeur d'architecture rurale, dont les soixante-douze fascicules sur la construction en pisé ont été diffusés dans le monde entier. Les projets-phares à l'horizon international nous ont été davantage évoqués en Allemagne, en Autriche ou en Suisse. Cela dit, de visites en revisites, la référence allemande à la «Baubiologie» ${ }^{(21)}$ comme entité signifiante d'une "architecture saine, utilisant des matériaux naturels [...] et souvent mue par une forte implication sociale» ${ }^{(22)}$ se fait moins explicite que les épreuves affrontées dans le développement des filières locales de matériaux géo-sourcés:

«Je me souviens que vous nous aviez accompagnés sur plusieurs chantiers de 2007 à 2008. Depuis, beaucoup de choses ont changé, la coopérative n'existe plus... Je suis auto-entrepreneur aujourd'hui. Je continue à faire du gros œuvre en terre mais cela reste compliqué... [...] Je ne sais pas si vous vous rappelez, il y avait une carrière dans la région. Un ingénieur allemand avait créé son exploitation et nous fournissait en matériaux, que ce soit de l'adobe ou des enduits de terre.... Vous l'aviez rencontré à l'époque, non? [Je hoche la tête en signe d'acquiescement.] Hé bien, son entreprise a fermé et le gars a quitté le pays! Les freins réglementaires et financiers n'ont pas été totalement levés en dix ans. Vous savez, le monde ne raisonne pas encore en coût global et c'est bien dommage... Quand on pense à toute l'énergie que le BTP dépense pour faire des bâtiments qui ensuite ne consommeront quasiment rien, on comprend tout de suite que l'enjeu c'est de développer les bio- et les géomatériaux! Au nord du département, un jeune couple s'est récemment lancé dans l'activité de fournisseurs de matériaux à base de terre crue mais le volume de leur production reste limité pour le gros œuvre. Du coup, moi, quand j'obtiens un chantier, je passe commande en Suisse germanophone. De l'autre côté de la frontière, il y a de véritables filières et leur essor permet de s'approvisionner sans trop de problème... Il suffit d'avoir une camionnette et de ne pas avoir peur de faire régulièrement des kilomètres!» ${ }^{(23)}$.

Sans tirer des conclusions précises sur l'intensité des échanges transfrontaliers, ce récit biographique poursuivi en 2018 avec un artisan français de la construction en terre témoigne de trajectoires non linéaires, accompagnées de mobilités centrifuges entre une zone de présence passée de minorités allemandes et des régions en relations historiques

19 Patrice Doat, Alain Hays, Hugo Houben, Silvia Matuk, François Vitoux, Construire en terre, Paris, Éditions Parenthèses, 1979.

20 Hubert Guillaud, «Une grande figure du patrimoine régional Rhône-Alpes: François Cointeraux (1740-1830): pionnier de la construction moderne en pisé», Carnets de l'architecture de terre, CRATerre-EAG, n 3 (1997); Laurent BARIdon, Jean-Philippe GARric et Gilbert Richaud (dir.), Les leçons de la terre. François Cointeraux (1740-1830). Professeur d'architecture rurale, Paris, INHA/Éditions des Cendres, 2016.

21 D’origine allemande, ce néologisme a été créé en 1969 par le professeur Anton Schneider, fondateur de l'Institut de Baubiologie et d'écologie de Neubeuern, en associant trois radicaux linguistiques: «Bau-» (maison, peau, chez-soi, tanière), «-bio-» (vie, force liée à la nature, au monde animé) et «-logie» (parole, science).

22 Dominique Gauzin-Müller, 25 maisons écologiques, Paris, Éditions du Moniteur, 2005, p. 8-17.

23 Extrait d'un entretien réalisé en février 2007 par Sophie Némoz auprès d'un auto-entrepreneur, âgé de 46 ans, maître d'œuvre et constructeur de gros œuvre en terre crue dans le Dauphiné. 
avec l'aire germanique. Dans les interstices, le truchement des interactions repose sur une action calculée au coup par coup autour des matériaux géo-sourcés. À cet égard, reprenant Michel de Certeau ${ }^{(24)}$, nous pourrions dire que ces mouvements liminaires relèvent de comportements tactiques, jouant avec les discontinuités spatiales, profitant et dépendant des occasions rencontrées de part et d'autre des frontières. D’après ce constructeur, les opportunités d'approvisionnement en terre se trouvent à proprement parler sur un «terrain qui lui est imposé tel que l'organise la loi d'une force étrangère » ${ }^{(25)}$. La comparaison internationale permet de «se défaire de l'apparente innocuité technique des instruments d'action publique de l'habitat durable " ${ }^{(26)}$. Après avoir étudié l'institutionnalisation d'un "format européen" et ses effets dans différents pays ${ }^{(27)}$, la morphologie du pouvoir a été éclairée à travers ses diverses ramifications nationales. «L'européanisation de l'habitat durable apparaît comme un changement d'architecture institutionnelle sans changement politique des rapports de forces et des représentations qui régissent les interactions entre l'habitat humain et son biotope " ${ }^{(28)}$. S'agissant de la terre, l'architecture la plus éminente se situe en Allemagne, à Weilburg an der Lahn, où un immeuble de sept étages construit en 1820 conserve un état impeccable. Après les années 1920, la technique de la terre allégée s'est diffusée dans l'aire germanique afin d'obtenir une meilleure isolation thermique à l'aide de matériaux au poids faible. Dans les périodes d'après-guerre que connaît l'Allemagne au $\mathrm{XX}^{\mathrm{e}}$ siècle, la pénurie de matériaux et d'ouvriers spécialisés suscite un intérêt renouvelé pour la construction en terre. Plusieurs milliers de lotissements ruraux sont ainsi bâtis en auto-construction. Le 4 octobre 1944, après bien des recherches scientifiques menées dans des laboratoires d'analyse des matériaux, un collectif d'experts, dont font partie Richard Niemeyer et Wilhelm Fauth, est à l'origine d'un décret publié au Journal officiel du Reich (Reichsgesetzblatt), intégrant le matériau géologique dans la réglementation du code de la construction afin de contribuer à un développement reconnu par tous en période de crise du logement ${ }^{(29)}$. Le franchissement des limites frontalières suppose un savoir et une appréciation de ce qui se produit en dehors des industries conventionnelles des composants français du bâtiment. L'analyse de ces productions en marge des centres institués du développement urbain durable invite à un déplacement du regard sur les situations d'articulation créative des connaissances et des matérialités par-delà les frontières de l'humanité.

\section{Engager les humanités environnementales sur la piste d'une pragmatique terrestre}

À l'heure où la terre suscite un regain d'attention des sciences humaines et sociales, insistant sur le caractère terrien de nos sociétés et ses enjeux politiques et conceptuels ${ }^{\left({ }^{(3)} \text {, }\right.}$

24 M. de Certeau, L'invention du quotidien (note 17), p. 60-61.

25 Ibid., p. 61.

26 S. NÉmoz, «L“'éco-logis” politique» (note 7), p. 41.

27 S. Némoz, L'«éco-logis»: une innovation durable (note 3); S. Némoz, «L'“éco-logis” politique» (note 7), p. 31-43.

28 S. NÉmoz, «L“éco-logis” politique» (note 7), p. 41.

29 Franz Volhard, Pierre Frey, Construire en terre allégée, Arles, Actes Sud, 2016.

30 Pierre Charbonnier, Bruno Latour, Baptiste Morizot, «Redécouvrir la terre», Tracés. Revue de Sciences humaines (en ligne), $\mathrm{n}^{\circ} 33$ (2017), p. 227-252, http://journals.openedition.org/traces/7071. 
les dimensions de sols et de territoires prédominent les cadres de pensée scientifique. Sous nos pieds se matérialiseraient la planète, le globe et ses défis environnementaux, sociaux et économiques. Si cette posture de surplomb a pu labourer les champs agraires et fonciers de la recherche, la terre demeure encore largement impensée en tant que matière première de l'habitat. La boue séchée, la terre battue, ou damée dans des coffrages dans le cas du pisé, ou encore le torchis, ou bien l'adobe, sont employés depuis des milliers d'années et toujours en œuvre dans pas moins d'un tiers des habitations humaines à travers le monde ${ }^{(31)}$. Ces techniques constructives bâtissent environ $15 \%$ du patrimoine rural français. Cela dit, ce matériau abondant, ne nécessitant aucune transformation chimique mais du temps et de la main-d'œuvre, est peu relié à la question terrestre, pourtant en plein essor dans les sciences de l'homme et tout particulièrement au sein des humanités environnementales qui placent les rapports collectifs à l'environnement naturel au centre de l'appréhension des mutations socio-politiques et d'une épistémologie transformée ${ }^{(32)}$.

Plutôt qu'une juxtaposition de savoirs disciplinaires, c'est une liminarité féconde que notre approche des phénomènes sociaux totaux recherche par la confrontation à une situation ancrée. En ce sens, nous proposons d'emprunter une piste «pragmatique» telle que les philosophes américains George Herbert Mead ${ }^{(33)}$ et John Dewey ${ }^{(34)}$ ont qualifié une méthode de pensée soucieuse de l'ensemble des implications pratiques. En articulant cette dernière à une démarche résolument inductive ${ }^{(35)}$, attentive au sens que les acteurs attribuent à leurs actions et aux catégories qu'ils mobilisent, notre travail de thèse a retenu comme définition de l'habitat durable celle de logements déclarés comme tels, que ce soit par leurs promoteurs institutionnels, les professionnels ou leurs habitants ${ }^{(36)}$. Notre engagement sur le terrain a précisément consisté en une ethnographie multi-située, une «ethnographie du/dans le monde» telle que Georges Marcus l’a théorisée plus tard: «Elle se déplace des sites uniques et des situations locales de la recherche ethnographique conventionnelle vers l'examen de la circulation des significations culturelles, des objets et des identités dans un espace-temps diffus » ${ }^{(37)}$. Des passerelles franco-germaniques ont ainsi été décelées dans la réalisation des projets de réhabilitation d'anciennes habitations rurales en région Auvergne-Rhône-Alpes. Elles s'étayent sur un renouvellement continu des tactiques des maîtres d'œuvre dont la situation française est plus précaire que dans les pays de langue allemande. Depuis les années 1980, le développement de filières locales de matériaux géo-sourcés est incertain en France ${ }^{(38)}$.

31 Romain Anger, Laetitia Fontaine, Bâtir en terre. Du grain de sable à l'architecture, Paris, Belin/Cité des sciences et de l'industrie, 2009, p. 8.

32 Guillaume Blanc, Élise Demeulenaere, Wolf Feuerhahn (éd.), Humanités environnementales. Enquêtes et contre-enquêtes, Paris, Publications de la Sorbonne (coll. Homme et société. Série Histoire environnementale), 2017.

33 George Herbert Mead, The Philosophy of the Act, Chicago, The University of Chicago Press, 1938.

34 John Dewey, Human Nature and Conduct, New York, Henri Holt and Company Editors, 1922.

35 Anselm Strauss, La trame de la négociation, Paris, L'Harmattan, 1991.

36 S. NÉmoz, L'«éco-logis»: une innovation durable (note 3).

37 Georges Marcus, «Ethnographie du/dans le système-monde. L'émergence d'une ethnographie multisituée», in: Daniel Cefä̈, L'engagement ethnographique, Paris, Éditions de l'École des hautes études en sciences sociales, 2010, p. 372.

38 Sophie Némoz, «Les mécanismes d'imbrication du patrimoine rural dans l'habitat durable. Sociologie d'un processus innovant entre différentes échelles territoriales », in : Nassima Dris (dir.), Patrimoines 
Il n'empêche des connexions cognitives, matérielles, sociales et symboliques qui contournent à la marge les contraintes des dispositifs nationaux de gouvernementalité. Bien que singuliers et situés en périphérie des grandes villes françaises, des logements en terre ont été rénovés ou construits durant ces trente dernières années.

De la relégation urbaine à la revitalisation de l'habitat en milieu rural, le mouvement de circulation des standards de la ville durable se poursuit aujourd'hui. Des traces ont été relevées plus récemment sur le terrain des communes des bassins de vie ruraux. Dans une autre approche comparative en région Bourgogne-FrancheComté, notre engagement ethnographique porte sur les logiques d'action locale tentant de construire des réponses à leurs difficultés (vacance des logements, fermetures de commerces et d'entreprises, vieillissement des habitants, isolement géographique, contraintes patrimoniales...). Les centres-bourgs sont désormais considérés comme un «enjeu majeur» de l'équilibre des territoires français. Cela s'exprime à travers un programme national expérimental mis en place en 2014 concernant la revitalisation de 54 d'entre eux.

À Salins-les-Bains où l'équipe municipale met en œuvre une série de dispositifs et de financements complexes dans le projet «Salins 2025 », la réception locale de ce programme volontariste d'attractivité résidentielle est observée depuis 2017 dans le cadre d'une recherche-action-formation ${ }^{(39)}$ que nous codirigeons. L'enquête en cours réunit des chercheurs ${ }^{(40)}$ de différentes disciplines de la Maison des sciences de l'Homme et de l'environnement Claude Nicolas Ledoux (USR 3124), des élus, des techniciens, des habitants de la collectivité et des usagers y travaillant mais résidant dans une autre commune. Après deux années d'expérimentation, les membres de l'équipe municipale ont sollicité «l'expertise d'universitaires pluridisciplinaires» sur un problème qu'ils appréhendaient comme extrêmement global et qu'ils ne parvenaient pas à résoudre dans le plan d'aménagement. Les opérations lancées en grand nombre, un peu partout sur la commune et en même temps, avaient su répondre aux différentes opportunités de financement de l'État, mais aussi des fonds européens, sans que leurs ajustements aux critères d'éligibilité, jugés «sectoriels» et «très concentrés sur la rénovation énergétique des logements», ne leur paraissent suffisants pour remédier au déclin du bourg. Telle qu'elle nous a été adressée, cette demande sociale renseigne d’une circulation étendue des standards de la ville durable au milieu rural. Une nouvelle phase la prolonge où nous avons été mis au défi de contribuer à un «accompagnement stratégique de Salins 2025 ».

et développement durable. Ressources - Enjeux - Lien social, Rennes, Presses universitaires de Rennes, 2012, p. 171-186.

39 Sophie NÉmoz, «Pistes pour une pragmatique terrestre: quels jardins pour dessiner quelles communautés?», Cinquième journée de l'atelier AC/DD «Habiter la transition. Des pratiques existantes aux politiques de transition: circulations et ambiguïtés », Maison des sciences de l'Homme et de l'environnement (MSHE Ledoux, USR 3124), Laboratoire de sociologie et d'anthropologie - Université de Franche-Comté (LaSA EA 3189), Équipe d’accueil «Architecture, Morphologie/Morphogénèse urbaine et Projets» (AMUP EA 7309) et la Région Bourgogne-Franche-Comté, Besançon, 12 octobre 2017.

40 L'équipe de recherche pluridisciplinaire adossée à la MSHE Ledoux est composée de Christian Guinchard et Sophie Némoz, sociologues (Laboratoire LASA), Alexandre Moine, géographe (Laboratoire ThéMA), Cyril Masselot, spécialiste en information et communication (Laboratoire CIMEOS), tous enseignants à l'Université de Franche-Comté, et de Laure Nuninger, chargée de recherche CNRS en archéologie spatiale (Laboratoire ChronoEnvironnement). 
Chez bien des groupes et des individus rencontrés localement depuis que nous travaillons sur ce projet, il y a l'impression d'une végétation qui "gagne du terrain » et qui «descend» sur la ville. Cette perception est associée à un sentiment de déclin du territoire urbain où l'«envahissement" ressenti semble être la trace d'une subordination de la culture par la nature. Le désappointement des habitants est manifeste dans cette sensation d'un bouleversement au cours duquel la culture ne domestique plus la nature. Cette impression est rattachée à un faisceau de signes comme la cessation d'activités commerçantes en centre-bourg, la fermeture d'entreprises, l'accroissement du transport routier en fond de vallée... Un peu plus en hauteur, la pousse des arbres vers le cœur de ville renvoie en contre-bas à des épreuves matérielles conséquentes. Courbés par les ruissellements, tapissés de végétaux et parfois fissurés, les murs et les escaliers présentent des aspérités dangereuses, des problèmes de sécurité pour les usagers et la commune (figure 3).

Figure 3. Prises de vues du centre-bourg en marchant avec d'autres enquêteurs
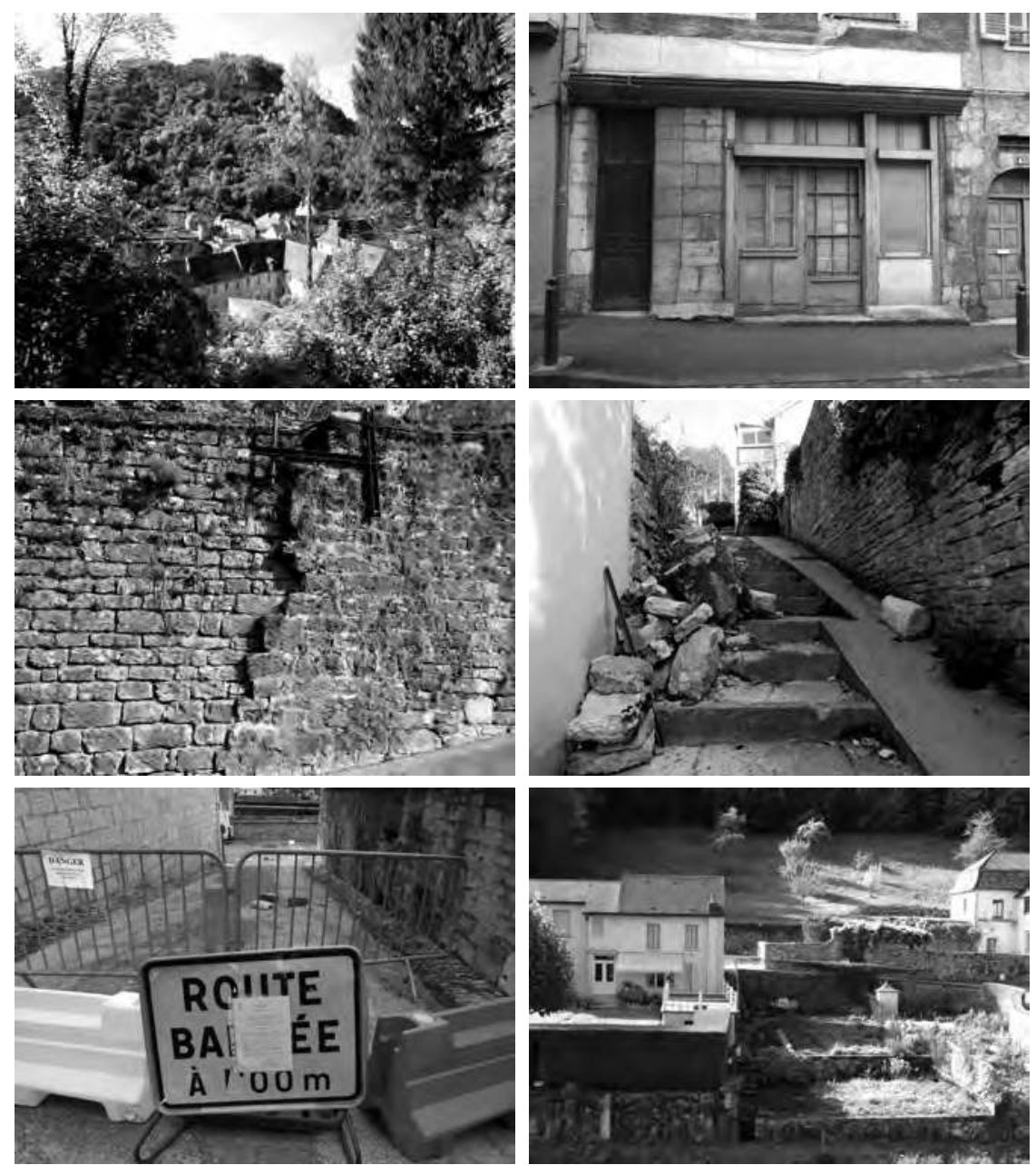

Sophie Némoz, 2017-2018 
La recherche de l'ensemble des implications pratiques s'appuie sur un régime de co-construction des connaissances, celui de l'«ingéniosité hétérogène» comme l'appelle la sociologie de la traduction(41). L'intervention des chercheurs consiste à inclure, à mettre ensemble et à articuler dans une perspective globale de développement les souhaits et les réflexions émanant de tous les acteurs de la ville, par l'intermédiaire d'une communauté d'enquêteurs. Constituée d'une cinquantaine de personnes, choisies en concertation pour leur représentativité et leurs dynamismes ${ }^{(42)}$, cette démarche vise une coopération où chacun dans sa sphère familiale, professionnelle et sociale, est en capacité de transmettre des informations et de constituer un relais auprès de la population dans son ensemble. Quelles perceptions et quelles pratiques a-t-elle de sa ville? Quelles attitudes sont partagées au cours d'un travail de construction en commun? Est-il possible d'en saisir toutes les implications et les imbrications? Cette réflexion a notamment porté sur les problèmes de sécurité imputés au manque d'entretien des murs des jardins-terrasses. Ils pourraient être la source de grandes difficultés si les actions sont dispersées, non coordonnées, ou au contraire le soubassement d'une stratégie de réhabilitation commune aux propriétaires et à la collectivité, valorisant le patrimoine, les parcelles communales et les jardins parfois en friches, situés à l'avant et/ou à l'arrière des maisons de ville. Les logements avec un petit terrain extérieur sont prisés des ménages souhaitant vivre dans la région selon les premiers résultats de l'enquête. Différenciée des tactiques, une action stratégique «[...] postule un lieu susceptible d'être circonscrit comme un propre et d'être la base d'où gérer les relations avec une extériorité de cibles ou de menaces » ${ }^{(43)}$. «La campagne autour de la ville», ainsi que «les objectifs et objets de la recherche», sont d'ailleurs mentionnés par Michel de Certeau comme des exemples où "toute rationalisation "stratégique" s'attache d'abord à distinguer d'un "environnement" un "propre", c'est-à-dire le lieu du pouvoir et du vouloir propres» ${ }^{(44)}$. Dans leurs accomplissements pratiques, il ne s'agit pas seulement d'une pluralité de savoirs disciplinaires mais aussi d'expertises techniques et de compétences d'entités très diverses. Les processus de totalisation en cours invitent les humanités environnementales à discerner les singularités afin d'associer leurs différentes réponses à la question collective des conditions de vie terrestre.

\section{Conclusion}

En partant de la poussière des éboulis d'un mur en pisé, au cours d'une réhabilitation de l'habitat en milieu rural, notre enquête ethnographique multi-située dans l'espace et dans le temps a fait connaissance avec des gestes tentant de transformer en ressources résidentielles et en atouts écologiques les propriétés vivantes de la terre.

41 Bruno Latour, Aramis ou l'amour des techniques, Paris, La Découverte, 1992.

42 «Un groupe d'accompagnement stratégique a d'abord été initié puis des sous-groupes de travail thématique ont progressivement émergé. Chaque groupe est composé d'élus de la ville, de techniciens, de chercheurs et de Salinois - Salinois étant entendu comme «usagers» de la ville: il peut s'agir d'habitants ou de personnes venant y travailler quotidiennement» (source: https://mshe.univ-fcomte.fr/ la-mshe-accompagne-salins-2025).

43 Michel de Certeau, L'invention du quotidien (note 17), p. 59.

44 Ibid. 
Si ce matériau millénaire que les initiés qualifient aujourd'hui de "géo-sourcé» est encore le plus répandu dans les enveloppes et les structures des habitations du monde, il est largement méconnu et les techniques constructives tendent à disparaître. On peut s'interroger sur ce qui fonde et relie ensemble le souci du gros œuvre de la terre chez des professionnels et des propriétaires d'anciennes fermes de la région AuvergneRhône-Alpes. Caractérisant ces collectifs variables à travers la durée et les territoires, leur attachement et leur détachement ont été constatés lors de situations diverses d'observation telles que des visites de chantiers, des ateliers des constructeurs, des conférences, des repas à la campagne, et des revisites en entreprises ou à domicile une décennie plus tard.

En questionnant tout autant les qualifications que les disqualifications des matériaux dits géo-sourcés, les revers, à commencer par des effondrements répétitifs parfois mortels, notre ancrage nous a permis d'aiguiser un regard réflexif et historique sur les coopérations de proximité qui se font et se défont par des connexions transfrontalières entre des êtres mouvants de manière relationnelle dans des systèmes sociaux entiers. Il est peut-être nécessaire de reconnaître la singularité des passerelles francogermaniques, cherchant à répondre aux difficultés de l'absence de filières de matériaux propres aux habitations en terre et à leur renouveau sur les sols français. Dans un espace-temps diffus, la construction, la déconstruction et la reconstruction de circuits de matérialités terreuses, mais aussi de personnes et autres vivants, d'énergies, de savoirs et de sens, s'étayent et s'effondrent selon des tactiques, au coup par coup, sans prévision, jouant avec les discontinuités spatiales et temporelles des dispositifs de gouvernementalité de l'habitat durable en Europe.

De la relégation urbaine à la revitalisation de l'habitat en milieu rural, l'étude de l'ensemble des implications pratiques de la terre est complexe. Ses multiples dimensions dans nos conditions de vie engagent les humanités environnementales à faire preuve d'une liminarité féconde entre des domaines généralement scindés par nos catégorisations. Le passage des frontières qu'elles tracent entre des savoirs très hétérogènes, et non seulement entre disciplines, met au défi d'un travail d'enquête collective, transdisciplinaire, afin de dissiper le trouble d'une vision morcelée entre nature et société et de bâtir le fond commun des circuits de connaissances d'une totalité dynamique plus vaste que les épreuves singulières.

\section{Résumé}

À partir d'une enquête ethnographique conduite de 2006 à 2018 dans des chantiers de rénovation et de construction de maisons individuelles en terre crue et en marge des grandes villes de la région Auvergne-Rhône-Alpes, ce texte propose d'examiner comment la circulation des échanges franco-germaniques, dont font preuve les acteurs du gros auvre, fonde l'ingéniosité et l'intelligibilité de leurs gestes singuliers. Après avoir présenté le cadre bâti et rural de la mise en cuvre actuelle d'un matériau millénaire, disponible mais diversement accessible selon les pays du monde, l'analyse de l'ensemble des implications pratiques montrera le caractère tactique des mouvements liminaires. Multiplier les points de vue, parfois antagonistes, dans un espace-temps diffus et au regard des humanités environnementales permet de repenser les phénomènes sociaux 
totaux et l'ensemble des implications pratiques entre des domaines généralement scindés par le dualisme entre nature et culture, entre sociétés et sciences.

\begin{abstract}
Based on an ethnographic survey conducted from 2006 until 2018 in building sites and renovation projects of detached houses with earth materials in the margins of the big cities in Auvergne-Rhone-Alpes region, the paper aims to examine how the circulation of Franco-German exchanges between actors in the main work builds the ingenuity and the intellibility of their singular gestures. After having presented the built and rural environment of current implementation of a building material for millennia, available but diversely accessible across national jurisdictions, the analysis shows the tactical dimension in the movements of liminality. The multiplication of perspectives, sometimes antagonistic, in a dispersed space-time and in terms of environmental humanities, enables to rethink the total social phenomena and the overall practical implications between fields generally divided by the dualism of nature and culture, societies and sciences.
\end{abstract}

